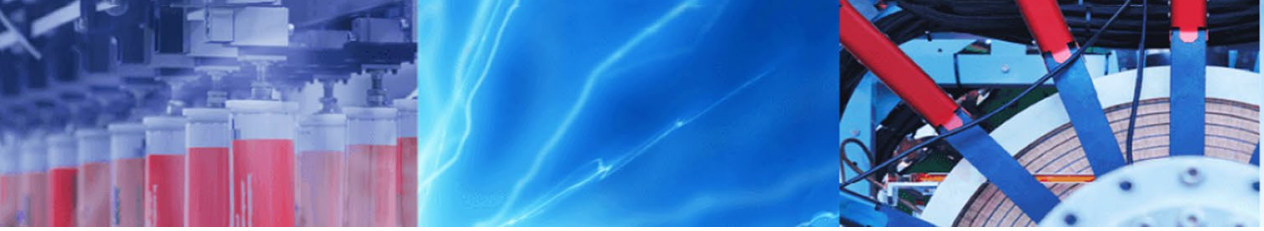

Research Article

\title{
Al-CNT-Ni composite with significantly increased strength and hardness
}

\author{
Md Muktadir Billah ${ }^{1,2} \mathbb{D} \cdot$ Quanfang Chen $^{2}$
}

(c) Springer Nature Switzerland AG 2019

\begin{abstract}
In this study, aluminium-carbon nanotube-nickel (AI-CNT-Ni) composite was prepared by powder metallurgy from pure Al powder and Ni-encapsulated CNT. Previously, carbon nanotubes were coated with nickel following ultraviolet-assisted electroless deposition method. Tensile strength and hardness of the composites were found to increase in proportion to the CNTs addition. For an addition of $7 \mathrm{wt}$.\% Ni-coated CNTs containing $2.5 \mathrm{wt} . \%$ nanotubes of 8-15 nm diameter (Ni to CNT ratio was 1-0.357), resultant tensile and yield strength were increased by $129.26 \%$ and $157.48 \%$ respectively compared to pure aluminium. Hardness was also increased by $171.39 \%$. These results were obtained while conventional sintering was followed by further consolidation by Hot Isostatic Pressing (HIP). Enhanced mechanical properties are attributed to the well dispersion of CNTs in aluminium matrix and strong interfacial bonding between CNT and aluminium. Well dispersion of CNTs and strong interfacial bonding was resulted from the uniform Ni-encapsulation as verified by red shift in Raman Spectroscopy.
\end{abstract}

Keywords $\mathrm{Al} / \mathrm{CNT}$ composite $\cdot \mathrm{Ni}$-encapsulation $\cdot$ Electroless deposition $\cdot$ Interfacial bonding $\cdot$ Raman spectroscopy

\section{Introduction}

Light and strong metallic materials with good electrical conductivity are greatly needed in automobiles and aerospace applications as well as in power grids (hanging lines). Aluminium is a traditional light metal conductor (density $2.7 \mathrm{~g} / \mathrm{cm}^{3}$ ) with the fourth best conductivity (only after silver, copper and gold). However, strength of pure aluminium is relatively low which is not sufficient for application like power transmission lines. Although alloying can significantly increase its strength, this results in simultaneous reduction in electrical conductivity. Therefore, other ways to increase the strength meanwhile without decreasing electrical conductivity is important. Recently carbon nanotubes (CNTs) have been added to aluminium due to CNT's exceptional mechanical, electrical and thermal properties $[1,2]$ including high young's modulus (1.0-1.8 TPa), high tensile strength (30-200 GPa) and high elongation at break (10-30\%) [3-6]. Moreover, CNT's thermal conductivity is comparable to that of diamond and electrical conductivity is like that of metals [7]. Thus, Al/CNT composites are considered as the novel attractive conductive materials due to their light weight coupled with potential high strength and high conductivity [8-12].

The ways of adding CNTs into aluminium include ball milling associated with powder metallurgy, mixing CNTs into molten aluminium and casting as well as high-pressure torsion etc. [12-15]. Based on published results all these methods are unable to disperse CNTs from bundles due to strong van der waals force in nanoscale range, therefore the resultant mechanical strength is not as high as expected and the resultant conductivity is lower than pure aluminium. Also, week interfacial bonding of CNT with aluminium matrix is of high concern.

$\triangle$ Md Muktadir Billah, mdmuktadirbillah@knights.ucf.edu | ${ }^{1}$ Materials and Metallurgical Engineering Department, Bangladesh University of Engineering and Technology, Dhaka, Bangladesh. ${ }^{2}$ Mechanical and Aerospace Engineering Department, University of Central Florida, 4000 Central Florida Blvd, Orlando, FL 32816-2450, USA. 
In this paper, authors have developed Al-CNT-Ni composite by powder metallurgy where nickel encapsulated multi-walled carbon nanotubes (MWCNTs) were mixed with pure aluminium powders. Resultant mechanical properties i.e. strength and hardness together with electrical conductivity were investigated for different diameter CNTs addition and different consolidation methods including Hot Isostatic Pressing (HIP). Nickel encapsulation was used to reduce the effect of strong van der waals force in nanoscale range and strengthen interfacial bonding of CNT with aluminium matrix.

\section{Experimental}

MWCNTs with purity greater than $95 \%$ were purchased from Cheap Tubes Inc. Two different diameter CNTs were used for this study: diameter between 30 and $50 \mathrm{~nm}$ with axial length between 10 and $20 \mu \mathrm{m}$ and diameter between 8 and $15 \mathrm{~nm}$ with axial length between 10 and $50 \mu \mathrm{m}$. CNTs were first purified to get rid of impurities such as amorphous carbon, metal catalyst and smaller fullerenes by ultrasonically dispersing in an aqueous solution of $\mathrm{HNO}_{3}(70 \%)$ at $80 \mathrm{C}$ for $2 \mathrm{~h}[16,17] . \mathrm{HNO}_{3}$ treatment also modifies surface properties to get better dispersion in the electrolyte with high-density activation sites for subsequent reactions [18]. After acid treatment CNTs become hydrophilic to adsorb metallic particles easily onto CNT's surface [18-20]. MWCNTs were then rinsed with deionized (DI) water until the solution was neutral.

Functionalized MWCNTs were then dispersed in an electroless nickel electrolyte for nickel encapsulation. In same amount of electrolyte almost double amount (weight) of smaller diameter CNTs were added compared to that of larger diameter CNTs. CNTs were first ultrasonically dispersed in the electrolyte solution under an ultra-violate (UV) exposure (a UV lamp of $100 \mathrm{~W}$ ) for $20 \mathrm{~min}$ (Fig. 1a). The UV exposure results in emission of electrons from valance band as free electrons on CNT's surface (Fig. 1b) and attract nickel ions in the electrolyte to the vicinity of CNTs due to coulombic force (Fig. 1c). $\mathrm{NaBH}_{4}$ solution was then added into the electrolyte to trigger $\mathrm{Ni}^{\mathrm{O}}$ atoms reduced on CNT's surface (Fig. 1d) [21]. After rinsing with DI water thoroughly the activated CNTs were dipped into a standard $\mathrm{Ni}$ electroless solution at $50 \mathrm{C}$ for $20 \mathrm{~min}$. Finally, the Ni-encapsulated CNTs were received (Fig. 1e) after being washed with DI water, filtered off and dried. Composition of the electroless nickel electrolyte is shown in Table 1. Ammonia solution was used to adjust $\mathrm{pH}$ to 8.5. Coating morphology of Ni-encapsulated CNTs was studied using Zeiss ULTRA-55 Field Emission Gun Scanning Electron Microscope (FEG-SEM), as shown in Fig. 2a. Electroless nickel deposition on CNTs without UV exposure was also carried out (Fig. 2b). It's evident that UV assisted Niencapsulation resulted in uniform nickel coating on CNT's surface.

$\mathrm{Ni}$-coated CNTs were then ultra-sonicated for $2 \mathrm{~h}$ in ethyl alcohol and after adding Al powders the mix was ultra-sonicated for another $2 \mathrm{~h}$. Following drying, the mixture of $\mathrm{Al}$ and $\mathrm{Ni}$-coated CNTs was pressed into pellet of $13 \mathrm{~mm}$ diameter under a unidirectional pressure of $400 \mathrm{MPa}$. Pure Al pellets were also prepared similarly. All the pellets were sintered in nitrogen atmosphere at $600 \mathrm{C}$ for $2 \mathrm{~h}$. HIP was also used for further consolidation of 8-15 nm diameter CNTs reinforced composites. HIP was conducted at $550 \mathrm{C}$ for $90 \mathrm{~min}$ at $200 \mathrm{MPa}$ pressure.

Archimedes principle was used to measure density using distilled water as an immersion medium. Effects of $\mathrm{Ni}$-coated CNTs addition on the resultant tensile strength and micro-hardness were investigated. Tensile specimens were prepared with rectangular cross-sectional area as per ASTM E8/E8M-09. Tensile test was conducted using Mechanical Testing and Simulation (MTS) Tytron Tester.

Table 1 Composition of Ni electrolyte

\begin{tabular}{ll}
\hline Chemicals & Concentration (g/L) \\
\hline $\mathrm{NiSO}_{4} \cdot 6 \mathrm{H}_{2} \mathrm{O}$ & 35 \\
$\mathrm{NaH}_{2} \mathrm{PO}_{2}$ & 35 \\
$\mathrm{C}_{6} \mathrm{H}_{5} \mathrm{Na}_{3} \mathrm{O}_{7}$ & 18 \\
$\mathrm{NH}_{3}$ solution & For $\mathrm{pH}$ adjustment \\
\hline
\end{tabular}

Fig. 1 Sketch of electrochemical deposition of nickel on CNTs stimulated by photon radiation, a photons radiation on CNTs, b electrons emitted on CNT's surface, $\mathbf{c}$ metal ions in electrolyte attracted to CNTs by coulombic force, $\mathbf{d}$ metal atoms reduced after reaction between ions and electrons and e Ni-encapsulated CNTs

\section{SN Applied Sciences

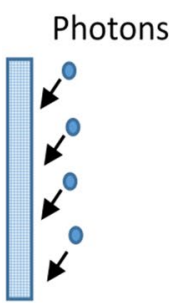

(a)

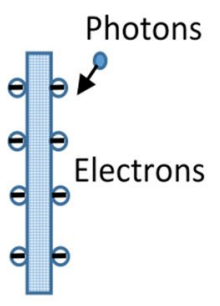

(b)

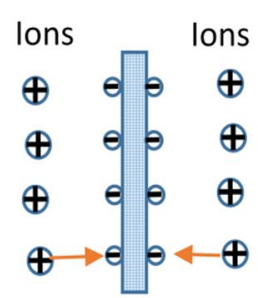

(c)

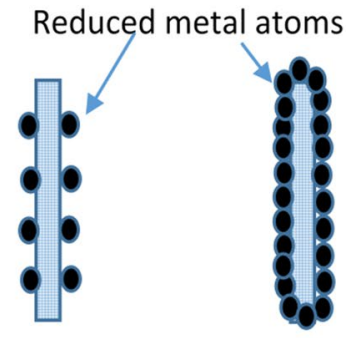

(d) (e) 

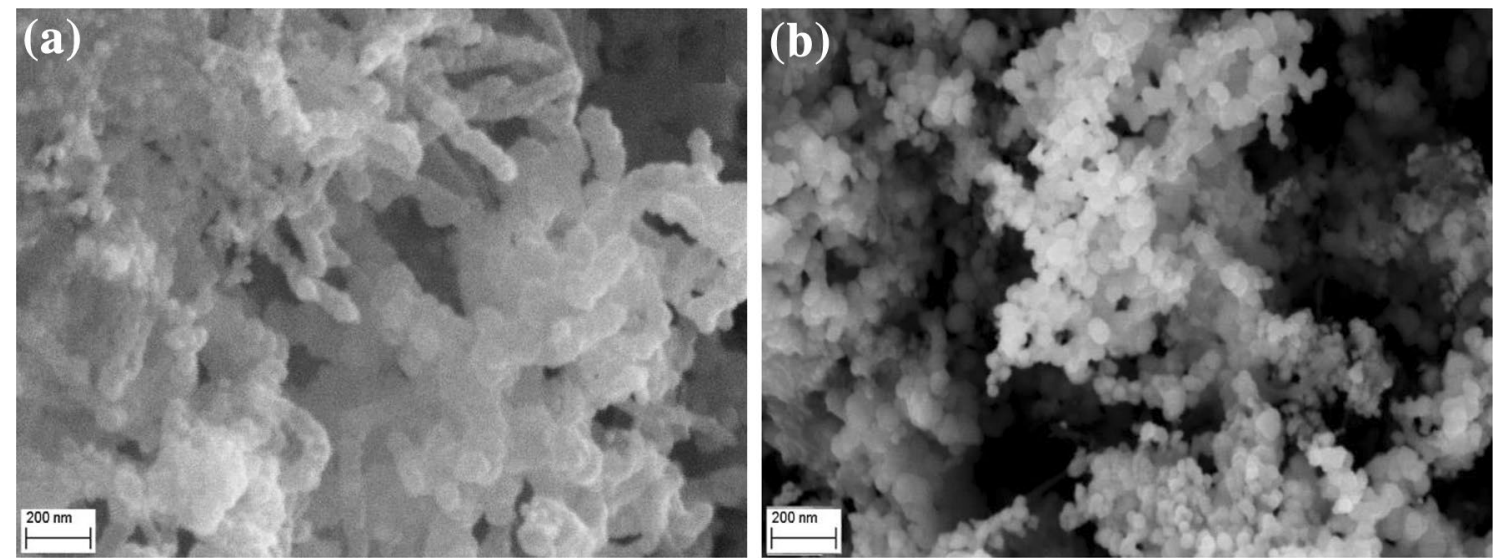

Fig. 2 Ni-encapsulated CNTs a with UV exposure and $\mathbf{b}$ without UV exposure

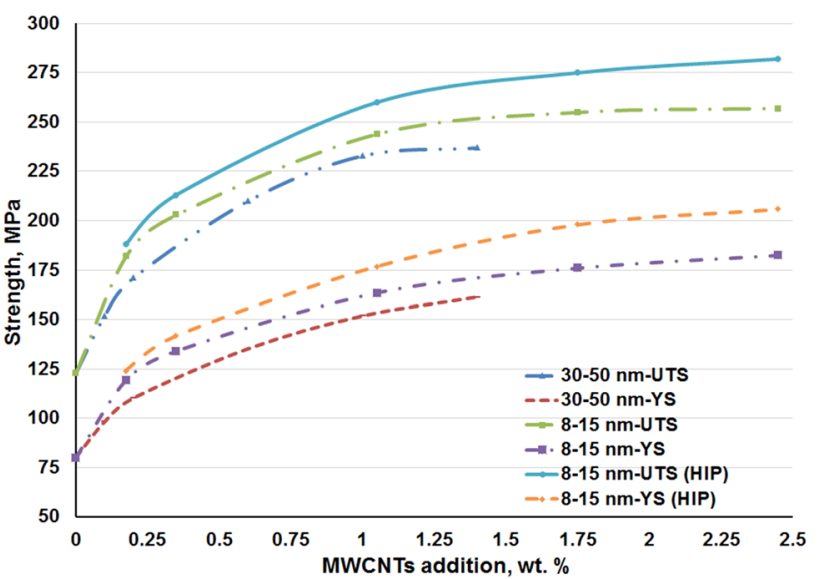

Fig. 3 Effect of CNTs addition on strength

Fracture surfaces were studied using Zeiss ULTRA-55 FEG-SEM. QV-1000 Vickers Hardness Tester was used to measure micro-hardness according to ASTM E-384 with an applied load of $50 \mathrm{~g}$-force for $30 \mathrm{~s}$. Diagonal lengths of the indentations were measured under SEM to calculate the hardness values.

\section{Results and discussion}

Figure 3 shows the effect of Ni-coated CNTs addition on both yield strength (YS) and ultimate tensile strength (UTS) of aluminium. CNTs coated with nickel under UV exposure (Fig. 2a) were only used to prepare the composites. A maximum of $7 \mathrm{wt} . \% \mathrm{Ni}$-coated CNTs (each of $30-50 \mathrm{~nm}$ and $8-15 \mathrm{~nm}$ diameter) was added in aluminium. Nickel to CNT ratio was $1-0.20$ for $30-50 \mathrm{~nm}$ diameter CNT and 1-0.357 for 8-15 nm diameter CNT. Thus, 7 wt.\% Ni-coated CNTs contain $1.4 \mathrm{wt} . \%$ actual CNTs when diameter of CNT is $30-50 \mathrm{~nm}$ (Al-1.4CNT-5.6Ni) and $2.5 \mathrm{wt} . \%$ actual CNTs when diameter of CNT is $8-15 \mathrm{~nm}$ (Al-2.5CNT-4.5Ni). For both type, addition of CNTs enhanced yield and ultimate tensile strength significantly. Strength was increased rapidly initially with addition of CNTs. Later, increase in strength was almost linearly proportional to any further addition. The smaller diameter CNTs resulted in greater strength values compared to the larger diameter ones. This is because for the same amount of CNTs addition, smaller diameter CNTs result in much finer inter particle distance and larger surface to volume ratio compared to that of the larger diameter CNTs. Later, HIP process was followed for 8-15 nm diameter CNTs reinforced composites. For Al-2.5CNT-4.5Ni composite (8-15 nm diameter) when conventional sintering was followed by HIP relative density was increased from 96.2 to $99.1 \%$. Due to the further consolidation with HIP following conventional sintering, further increase in both yield and ultimate tensile strength were observed (Fig. 3). Compared to pure aluminium, YS and UTS of Al-CNT composites were increased maximum by $157.48 \%$ and $129.26 \%$ respectively for $\mathrm{Al}-2.5 \mathrm{CNT}-4.5 \mathrm{Ni}$ composite (8-15 nm diameter) when conventional sintering was followed by HIP.

Figure 4 shows that micro hardness also increased continuously with increasing CNTs addition. Similar to tensile properties, micro hardness also increased rapidly with initial addition of CNTs. Later, with further addition hardness increased slowly. Compared to pure aluminium, micro hardness was increased maximum by $171.39 \%$ for $\mathrm{Al}-2.5 \mathrm{CNT}-4.5 \mathrm{Ni}$ composite $(8-15 \mathrm{~nm}$ diameter) when conventional sintering was followed by HIP.

The fracture surface of pure Al and Al-CNT-Ni composites are shown in Fig. 5. Dimples on fracture surface indicates the ductile fracture mode of pure Al (Fig. 5a). At fracture surface of the $\mathrm{Al}-0.6 \mathrm{CNT}-2.4 \mathrm{Ni}$ composite (30-50 $\mathrm{nm}$ diameter), CNTs were observed to be 
Fig. 4 Effect of CNTs addition on VH

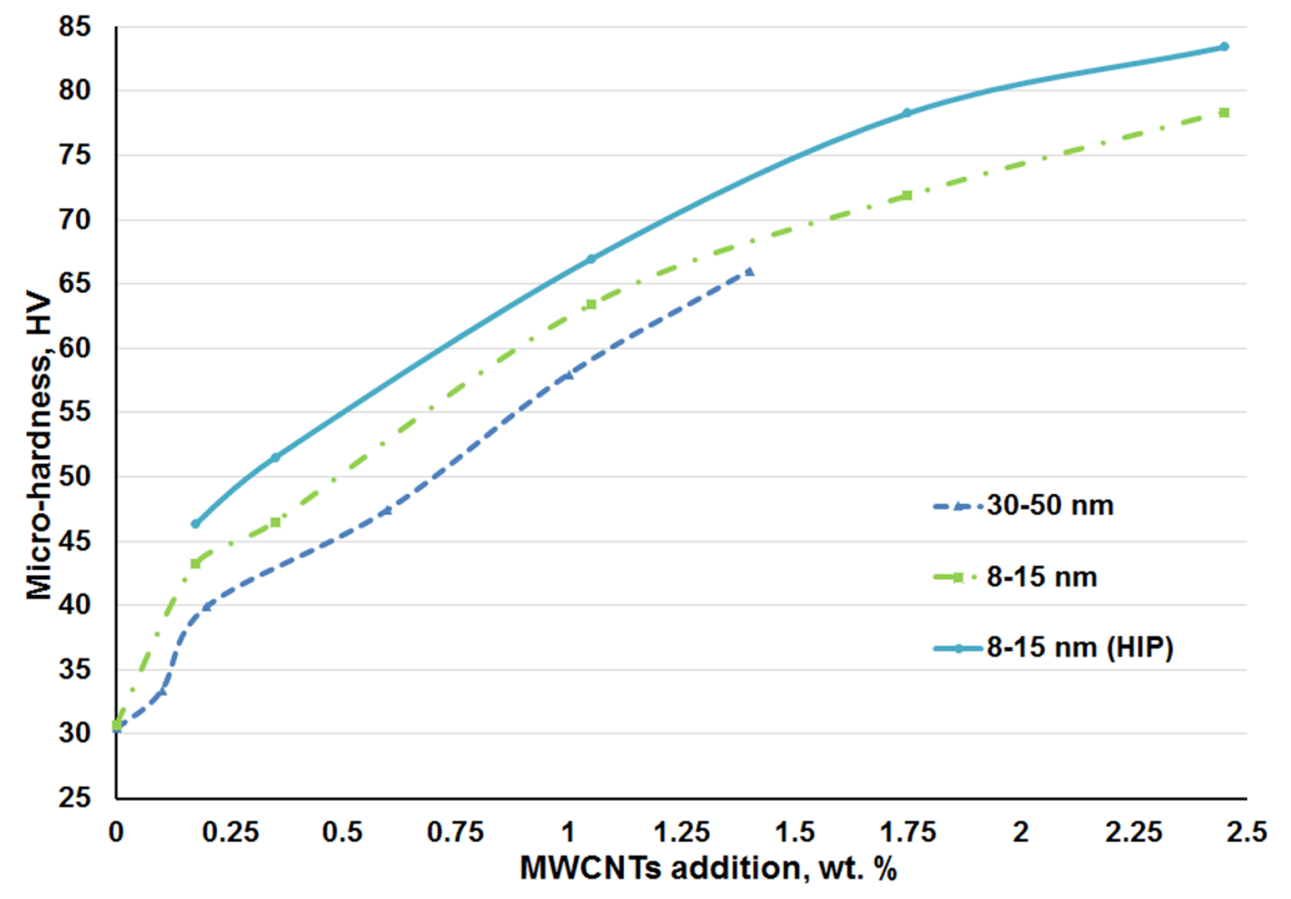

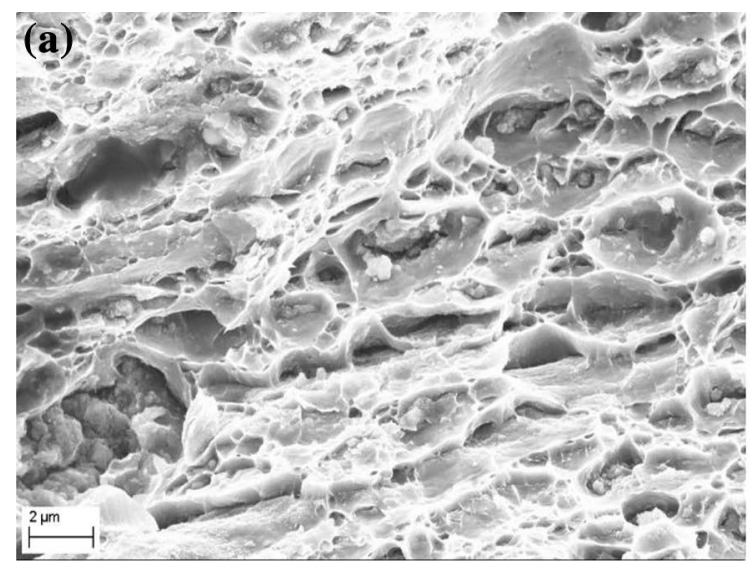

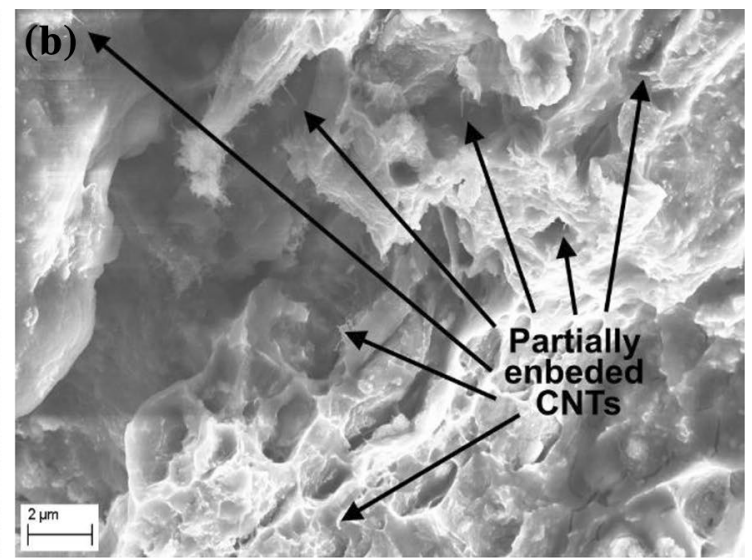

Fig. 5 SEM micrographs of fractured surfaces of $\mathbf{a}$ pure Al and $\mathbf{b}$ Al-0.6CNT-2.4Ni composite (30-50 nm diameter)

embedded partially in the Al matrix with short pull-out length (Fig. 5b). It's also evident that the Ni-encapsulated MWCNTs get dispersed well in the Al matrix. Ni coating prevents direct contact between CNTs by reducing van der waals force on CNT's surface and thus results in better CNT dispersion as well as better interfacial bonding between CNT and aluminium matrix. These results in the enhanced mechanical properties of the composites. In addition, the encapsulated nickel as shown in Fig. 5b appears in a chain of balls that forms "nailing" effects to increase the load bearing capability to prevent CNTs from withdrawing out. This indicates the strong interfacial bonding between Al matrix and CNT resulted from the uniform Ni-encapsulation [20]. Strong interfacial bonding results in partial embedment of CNTs in the matrix and short pull-out lengths.

Raman Spectroscopy was conducted for pristine CNTs, CNTs coated with nickel without UV exposure and CNTs coated with nickel with UV exposure (Fig. 6). It shows that after $\mathrm{Ni}$-coating both $\mathrm{D}$ band and $\mathrm{G}$ band are shifted to the left. For Ni-encapsulation under UV exposure both band shifted further to the left. These results indicate the formation of strong interfacial bonding between CNT and $\mathrm{Ni}$ for UV-assisted $\mathrm{Ni}$-encapsulation. $\mathrm{D}$ band is correspond to the distortion of $\mathrm{SP}^{2}$ structure and G-band arises from the stretching of the $\mathrm{C}-\mathrm{C}$ bond. When these bands shows red shift (towards lower frequency), it indicates strong C-metallic atom bond formation [23]. Later, $\mathrm{Ni}$ 
Fig. 6 Raman spectroscopy (red shift)

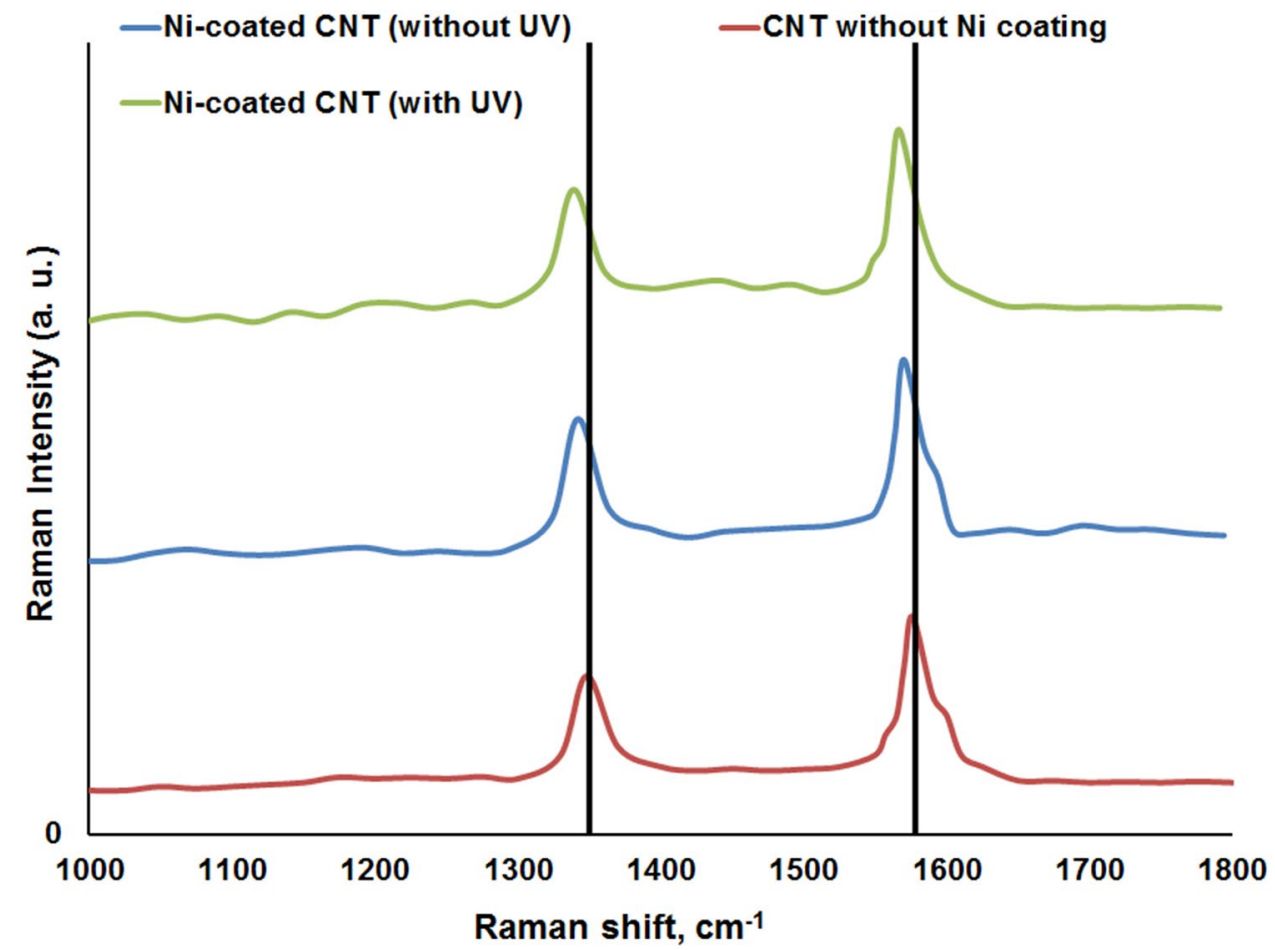

easily form metallic bond with aluminium matrix which is much stronger than CNT-Al (metal-non-metal) interfacial bonding.

In addition to the mechanical properties, resultant electrical conductivity of the Al-CNT-Ni composites was also investigated by using four-probe method (Fig. 7). Electrical conductivity was increased with addition of CNTs. Compared to pure aluminium, electrical conductivity was increased maximum to $112.5 \%$ for Al-2.5CNT-4.5Ni composite (8-15 nm diameter) when conventional sintering was followed by HIP. The increased electrical conductivity also implies a better interfacial bonding between CNT and Al matrix through uniform nickel encapsulation.
Fig. 7 Effect of CNTs addition on conductivity

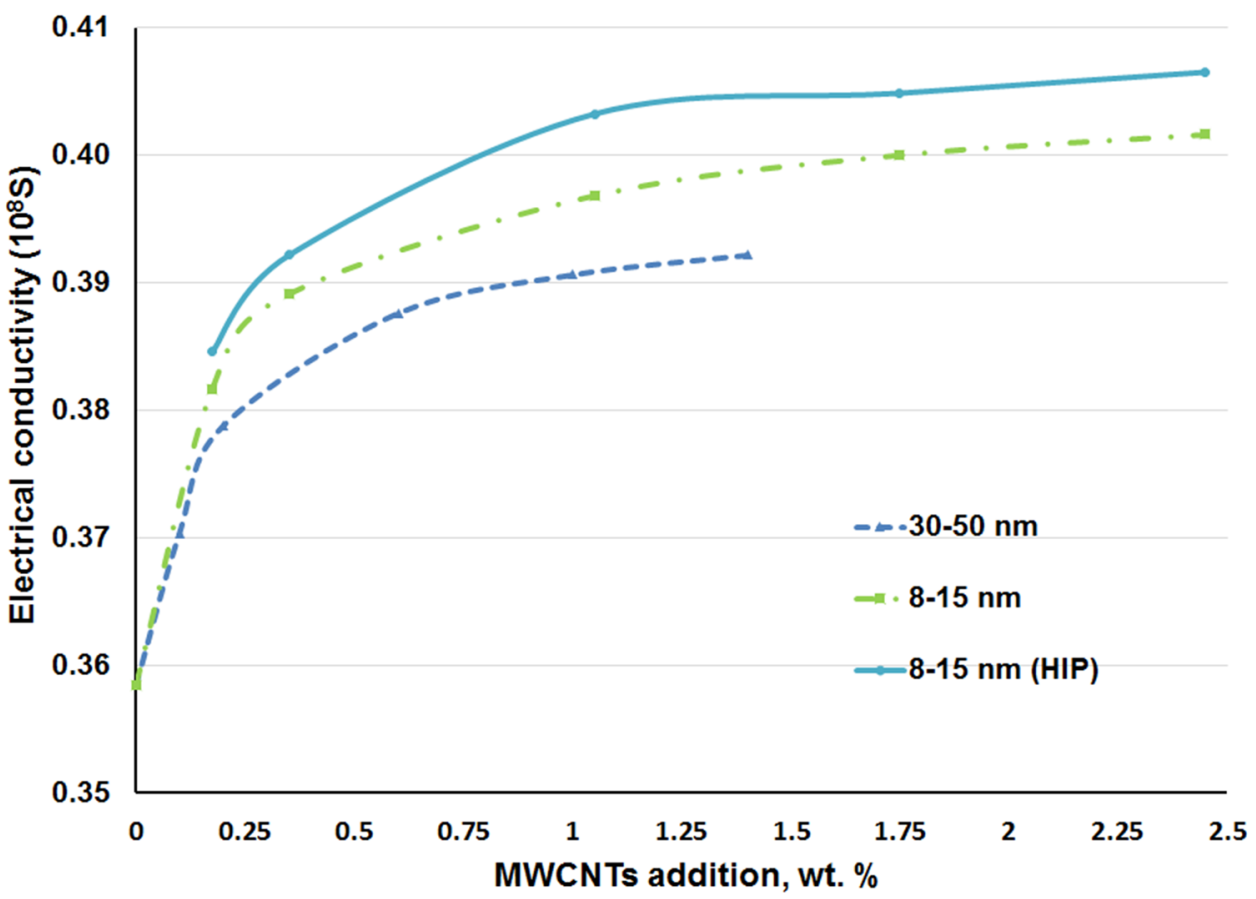

SN Applied Sciences 
Increase in tensile and yield strength of aluminium by CNTs reinforcement is reported in many studies so far. However, in most cases increase in YS strength was in the range of $110-160 \%$ and UTS in the range of $100-150 \%$ compared to pure aluminium [4-7]. In this context current study reported strength enhancement in the high end. However, the achievement of this study is not only enhancing the mechanical properties, rather to enhance electrical conductivity simultaneously unlike many other studies [22-25]. In most cases electrical conductivity was reported to drop with CNTs addition [22, 23].

\section{Conclusions}

Al-CNT-Ni composite was prepared by powder metallurgy method in which $\mathrm{Ni}$-encapsulated CNTs were mixed with pure aluminium powder. Resultant yield strength and ultimate tensile strength of the composites were enhanced significantly. For Al-2.5CNT-4.5Ni composite $(8-15 \mathrm{~nm}$ diameter) when conventional sintering was followed by HIP, yield strength and ultimate tensile strength were increased maximum by $157.48 \%$ and $129.26 \%$ respectively. Micro hardness was also increased by of 171.39 . More importantly, electrical conductivity was increased to $112.5 \%$ compared to that of pure aluminium. All these results are attributed to the well dispersed CNTs and the strong interfacial bonding resulted from uniform $\mathrm{Ni}$ encapsulation on CNT's surface. Uniform nickel encapsulation was achieved by UV-assisted electroless deposition method. The good interfacial bonding was verified by red shift in Raman spectrometry.

Acknowledgements The work was supported by the National Science Foundation and the Department of Energy.

\section{Compliance with ethical standards}

Conflict of interest The authors declare that they have no conflict of interest.

\section{References}

1. Harris PJF (2004) Carbon nanotube composites. Int Mater Rev 49(1):31-43

2. Curtin WA, Sheldon BW (2004) CNT-reinforced ceramics and metals. Mater Today 7(11):44-49

3. Desai AV, Haque MA (2005) Mechanic of the interface for carbon nanotube-polymr composites. Thin Wall Struct 43(11):1787-1803

4. Lau AK, Hui D (2002) The revolutionary creation of new advanced materials-carbon nanotube composites. Compos B Eng 33(4):263-277

5. Treacy MM, Ebbesen TW, Gibson JM (1996) Exceptionally high young's modulus observed for individual carbon nanotubes. Nature 381(6584):678-680
6. Wong EW, Sheehan PE, Lieber CM (1997) Nanobeam mechanics: elasticity, strength, and toughness of nanorods and nanotubes. Science 277(5334):1971-1975

7. Berber S, Kwon Y, Tomanek D (2000) Unusually high thermal conductivity of carbon nanotubes. Phys Rev Lett 84:4613-4616

8. Carreno-Morelli E (2006) Carbon nanotube-metal matrix composites, the Dekker encyclopedia of nanoscience and nanotechnology. Taylor and Francis, New York, pp 1-9

9. Torralba JM, da Costa CE, Velasco F (2003) P/M aluminum matrix composites: an overview. J Mater Process Technol 133(1-2):203-206

10. Fogagnolo JB, Velasco F, Robert MH, Torralba JM (2003) Effect of mechanical alloying on the morphology, microstructure and properties of aluminium matrix composite powders. Mater Sci Eng A 342(1-2):131-143

11. Moons T, Ratchev P, Smet PD, Verlinden B, Houtte PV (1996) A comparative study of two Al-Mg-Si alloys for automotive applications. Scripta Mater 35(8):939-945

12. Shadakshari R, Mahesha K, Niranjan HB (2012) Carbon nanotube reinforced aluminium matrix composites-a review. Int J Innov Res Sci Eng Technol 1(2):206-213

13. Esawi AMK, Morsi K, Sayed A, Taher M, Lanka S (2010) Effect of carbon nanotube (CNT) content on the mechanical properties of CNT-reinforced aluminium composites. Compos Sci Technol 70(16):2237-2241

14. Liao JZ, Tan MJ, Sridhar I (2010) Spark plasma sintered multi-wall carbon nanotube reinforced aluminum matrix composites. Mater Des 31(1):96-100

15. Esawi AMK, Morsi K (2007) Dispersion of carbon nanotubes (CNTs) in aluminum powder. Compos A Appl Sci Manuf 38(2):646-650

16. Maqbool A, Khalid FA, Hussain MA, Bakhsh N (2014) Synthesis of copper coated carbon nanotubes for aluminium matrix composites. IOP Conf Ser Mater Sci Eng 60(1):012040

17. Peng YT, Hu YZ, Wang H (2007) Tribological behaviors of surfactant-functionalized carbon nanotubes as lubricant additive in water. Tribol Lett 25(3):247-253

18. Zhao B, Yadian BL, Li ZJ, Liu P, Zhang YF (2009) Improvement on wettability between carbon nanotubes and Sn. Surf Eng 25(1):31-35

19. Jagannatham M, Sankaran S, Prathap H (2015) Electroless nickel plating of arc discharge synthesized carbon nanotubes for metal matrix composites. Appl Surf Sci 324:475-481

20. Li Q, Fan S, Han W, Sun C, Liang W (1997) Coating of carbon nanotube with nickel by electroless plating method. J Appl Phys 36B:501-503

21. Yan T, Li L, Wang $L$ (2012) A simple nickel activation process for electroless nickel-phosphorus plating on carbon fiber. BioResources 8(1):340-349

22. Laha T, Liu Y, Agarwal A (2007) Carbon nanotube reinforced aluminum nanocomposite via plasma and high velocity oxy-fuel spray forming. J Nanosci Nanotechnol 7:1-10

23. Liu ZY, Xiao BL, Wang WG, Ma ZY (2014) Tensile strength and electrical conductivity of carbon nanotube reinforced aluminum matrix composites fabricated by powder metallurgy combined with friction stir processing. J Mater Sci Technol 30(7):649-655

24. Liu ZY, Xiao BL, Wang WG, Ma ZY (2012) Singly dispersed carbon nanotube/aluminum composites fabricated by powder metallurgy combined with friction stir processing. Carbon 50:1843-1852

25. Nam DH, Cha SI, Lim BK, Park HM, Han DS, Hong SH (2012) Synergistic strengthening by load transfer mechanism and grain refinement of CNT/AI-Cu composites. Carbon 50:2417-2423

Publisher's Note Springer Nature remains neutral with regard to jurisdictional claims in published maps and institutional affiliations. 